\title{
An Evaluation of Product Identification Techniques for Mobile Phones
}

\author{
Felix von Reischach ${ }^{1,2}$, Florian Michahelles ${ }^{1}$, Dominique Guinard ${ }^{1,2}$, \\ Robert Adelmann ${ }^{1}$, Elgar Fleisch ${ }^{1,3}$, and Albrecht Schmidt ${ }^{4}$ \\ ${ }^{1}$ ETH Zürich, Switzerland \\ ${ }^{2}$ SAP Research, Switzerland \\ ${ }^{3}$ University of St. Gallen, Switzerland \\ ${ }^{4}$ University of Duisburg-Essen, Germany \\ \{freischach, fmichahelles, dguinard, efleisch\}@ethz.ch, \\ robert.adelmann@inf.ethz.ch, albrecht.schmidtaicb.uni-due.de
}

\begin{abstract}
Among others, consumer products can be purchased in the Internet and in traditional stores. Each of the two has dedicated advantages. An online survey conducted within the frames of this work investigates these advantages. It motivates the transition of the advantages of online shopping, such as access to recommendations of other consumers, to the sales floor. Recent trends in mobile phone technology, for example the emergence of the mobile Internet, enable exactly this transition, potentially enriching the shopping experience in the real world. A key challenge though is a fast and convenient identification of products. This work compares five product identification modalities for mobile phones in a comparative study. The dependent variables evaluated are 'task completion time' and 'perceived ease of use'. Our study is the first that quantifies the advantage of automatic identification. The results indicate that automatically identifying a product scanning a tag can be up to eight times faster than entering a product name in a text field. Surprisingly, barcode recognition using a camera phone can be conducted almost as fast and convenient as scanning an RFID tag. Our work provides a benchmark for developers having to choose appropriate identification technology for their mobile application.
\end{abstract}

\section{Introduction}

Over the past years, the Internet has developed into a valuable tool for consumers searching for unbiased and extensive product information. With the goal to take a rational buying decision, consumers frequently access price comparisons, technical specifications, consumer reviews, or similar information in the web. Online shops utilize the availability of this information and integrate according functionalities into their websites, creating a better experience [1] and positively influencing sales [2]. But although revenues in online shops are increasing, physical stores still retain their attractiveness to customers. A reason for that is certainly that the overall shopping experience created in a shopping mall or a corner store is very different from shopping with a web browser. 
The separation between products and related information, as mentioned above, leads to a phenomenon called "hybrid shopping" or "multi-channel shopping" [3]. As consumers want to leverage the transparency in the Internet and the shopping experience from their usual shopping, people go back and forth between searching for information on the Web and buying their products in real world shops. This leads to a fragmentation of the shopping process in time and space [4]. However, recent technology allows a time-wise and location-wise combination of Internet-based information and traditional shopping: Newer generations of handsets make the Internet portable, implicating that consumers can potentially access product-related information on the sales floor. When actually shopping, they can enjoy the advantages of both worlds: touch and see the products, while accessing a rich pool of product-related information. But although mobile Internet access is on the rise, product-related services have not been widely adopted by consumers yet. It seems that for their widespread diffusion a number of shortcomings regarding their usability have to be tackled. Among them are the limited possibilities to browse websites due to rather small displays and the cumbersome entry of text. Especially when in the context of shopping, the entry of longer text strings such as product name or product number seems to prevent people from accessing additional product information using their mobile device.

As an approach to this, research and practice have been investigating interaction techniques that facilitate the identification of products through mobile phones. On the one hand, mobile phones have been turned into barcode readers [5, 6]. Using the phone's camera and applying image recognition algorithms, common 1-dimensional barcodes as well as 2-dimensional barcodes can be identified. On the other hand, there are two technologies that turn mobile phones into readers of Radio Frequency Identification (RFID) tags. The first technology, Near Field Communication (NFC) [7], is a consumer-oriented transmission standard that allows reading of RFID tags by touching them with a mobile phone's antenna. Typical current NFC applications include mobile payment [8], smart posters [9] and ticketing [10]. Secondly, readers have been integrated into mobile phones that allow the identification of RFID tags at ultra high frequency $(860-960 \mathrm{MHz})$ from a distance up to $50 \mathrm{~cm}$ [11]. This so called Electronic Product Code (EPC) standard [12] is typically applied for supply chain applications and is in discussion to replace the barcode on consumer products in the future [13]. It is not yet clear though whether NFC or EPC will succeed in the consumer goods industry [14].

Yet there have been several projects from both research $[15,16,17,18]$ and business practice ${ }^{1}$ that take advantage of these new technical possibilities in the shopping context. However, to the best of our knowledge, none of the works has investigated the advantage of automatic identification compared to manual entry. And moreover, no work has focused on finding suitable product identification techniques for mobile shopping assistance. We investigate:

- How significant is the advantage of automatic product identification versus manual product identification?

- What is the most appropriate mobile identification technique for shopping applications?

\footnotetext{
${ }^{1}$ Among the first commercial mobile shopping assistants making use of automatic product identification techniques are CompareEverywhere, Scanlife, and Barcoo.
} 
To tackle these questions we proceed as follows: Firstly, we distinguish from related literature. Secondly, we motivate our experiment with an online-survey that aims at investigating the potential of mobile product-related services in general. Thirdly, we describe the design and execution of a user study conducted with 17 participants. Fourthly, we present the results of this study. We conclude interpreting these results and giving guidelines for developers.

\section{Related Work}

This section links our survey to related literature and distinguishes our main contribution, the user study, from existing works.

In the early 2000s, the "hype-phase" of e-commerce, a number of studies and surveys were performed that investigate the behavior of consumers when shopping online. These are predominantly settled in the areas of business research, or more specifically, around the terms marketing, consumer research, and decision making theories. Häubl and Trifts [19], for example, have investigated the effects of interactive decision aids on online shopper's buying behavior. Another relevant work has been performed by Ward and Morganowsky [4], examining how the availability of marketing information channels (Internet, print, broadcast, catalog) affects the channel for purchasing the good. They propose a better management of this so called 'channel confilict'. More specifically, Senecal and Nantel [2] investigate the usage of online recommendation sources through consumers and their influence on online product choices. Our survey differentiates from this by collecting generating up-todate data on what values consumers attribute to online shopping and shopping in traditional stores, what information sources they consult, and what they are willing to pay for product information on mobile phones.

When accessing product-related information on the mobile phone while shopping, a key requirement is a fast and convenient identification of products. Numerous researchers have suggested that mobile phones may be the ideal candidates for enriching real-world interactions with information from the Internet. We build up upon Ballagas et al. [20], who have explored and classified various mobile input modalities. Our work goes beyond this by comparing these modalities in a quantitative manner. In this, our approach is similar to the work of Broll et al. [21] as well as to the study of Rukzio et al. [22]. Both analyze and compare input techniques for interacting with the physical world. According to these studies, touching (e.g. NFC) and pointing (e.g. barcode identification, image recognition and EPC Tag Scan to some extent) are the preferred techniques if the object in question is within the reach of the user. We leveraged these results in our selection of modalities to be evaluated.

Our contribution extends the state of the art in two ways. Firstly, we specifically contribute a quantification of the advantage of automatic product identification techniques on mobile phones. While other studies have found out that there is an advantage in automatic identification on mobile phones, we aim at specifically determining: how big is this advantage? Secondly, our study aims at testing mobile identification techniques specifically for shopping applications. This is, for example, reflected in task 2, where the identification speed for several items is measured simulating a product comparison. 


\section{A Survey on the Potential of Mobile Product Information}

In order to investigate the potential of mobile-Internet-enhanced shopping, we conducted a web-based survey. The questionnaire consisted of 20 questions and took about 20 minutes to complete. The participants were recruited via email (friends, colleagues, friends of friends, and RFID researchers). We received 136 complete responses ( $25 \%$ females) of people with mixed backgrounds from 15 countries on 4 continents. The age range was between 18 and 50+. The educational levels of the participants were heterogeneous, with $88 \%$ of the participants having a university degree or higher. The language of the survey was English. In the following, we describe selected results from a descriptive evaluation of the survey.

The survey investigates the shopping behavior for three types of products: digital cameras, books, and wine. In the selection, we build up on the work of Nelson [23] and King and Balasubramanian [24], who distinguish between so called 'search products' and 'experience products'. Search products are dominated by properties that can be evaluated prior purchase, such as the 'amount of megapixels' when looking for a digital camera. In contrast, experience goods are dominated by attributes that cannot be known until purchase and use of the product: an example is wine. A book is somewhere in between. It can be browsed, but hardly read and assessed completely before purchase. This classification influences the way we buy products, especially the information we base our buying decision on.

The first part of the survey investigates the following question: What are the advantages and disadvantages of buying products online vs. in a real shop? We asked this question for all three of the above product types. The participants answered in free text. The responses entered show that a large majority of people sees, among others, roughly the following advantages for physical shopping (numbers refer to the purchase of a digital camera, but were similar for all products; similar answers were grouped manually):

- $\quad$ you can touch and feel the product (64\%)

- you can come back to the store in case of a problem with the product (57\%)

- you have the product immediately / no shipping required (15\%)

For online-shops the consumers reported the following benefits:

- $\quad$ easy price and product comparison and hence getting a better deal (60\%)

- $\quad$ easy access to technical details, in-depth reviews and customer comments $(52 \%)$

- convenience of not having to go shopping in person $(29 \%)$

Looking at the advantages of online-shopping it becomes clear that two important benefits are based on the access to additional information. We wanted to know, whether people actually mix searching for product information in the Web and shopping in traditional stores. We asked: Have you ever searched for product information in the Internet, but finally purchased the product in a real store? Of all participants, $79 \%$ were positive. Asked for the products searched for, electronic devices and books were among the ones stated the most. 
In order to get hints on what information consumers would appreciate on their mobile phone when shopping, we asked: On what information will you base your buying decision? Possible answers were: own experience, recommendations of friends and family members, technical features, visible features, commercial recommendations, price, recommendations of unknown Internet users, and independent consumer organizations and magazines. The answers differ significantly for each considered product type. When buying a digital camera, consumers predominantly rely on technical details (93\%), price $(85 \%)$ and recommendations of friends and family members (72\%). When shopping for books, the own experience (86\%) and recommendations of friends and family members $(84 \%)$ is what counts by far the most. When shopping for wine, people rely predominantly on their own experience $(87 \%)$, recommendations of friends and family members $(64 \%)$ and price $(64 \%)$. It seems that empowering users to share recommendations among friends and family members on the mobile phone provides a vast potential, especially for experience products.

Based on the preceding questions, indicating that mobile shopping applications have the potential to support consumers, we did a first step towards an assessment of their business potential and asked: How much would you pay for a rich collection of information about the product on your mobile phone in a real store (all three product categories)? The willingness to pay is similar among all products (digital camera, book, wine). For a digital camera, for example, the opinions are distributed as follows (selection from predefined answers):

- Nothing. This service should be generally free of charge (51\%).

- Nothing. But I would accept advertisements (18\%).

- I would be willing to pay roughly the value of a text message (19\%).

- I could imagine to pay between 3 and 5 dollars (5\%)

Some participants used the additional free text field we provided and entered other ways for compensation. Many proposed a flat rate tariff, others proposed to earn community credits though self-created recommendations and use these to pay, again others insisted the price should be relative to the item on hand.

Summarizing the survey results it becomes clear that people appreciate product information on their mobile phone. However, the product information must be easily accessible, which is not the case today. Besides mobile Internet flat rates and appropriate displays, easy identification is a fundamental element of the design of systems providing product information on mobile phones. This finding motivated our user study, which is described in the next section.

\section{Usability Study: Design and Execution}

The study consisted of two tasks and a follow-up interview. In the first task, the participants identified single products in a mixed sequence of five different identification techniques. In the second task the users simulated the comparison of three given products, identifying three products in a row. For time reasons we compared only the expectedly fastest manual identification technique to the expectedly fastest automatic identification technique. In the adjacent interview the participants rated each of the identification techniques regarding their 'perceived ease of use'. 


\subsection{Participants}

We recruited 17 participants (6 females) for the study. Their average age was 27.5 years with mixed professional backgrounds (secretary staff, physicians, business people, IT people, engineers, and others). All participants owned a mobile phone and were familiar with text input on mobile devices. The study was executed on two consecutive days in September 2008.

\subsection{Study Design}

We utilized a within-subject study design. The dependent variable was the time required to identify a product (task completion time). In the experiment we used 4 different wine bottles (denoted as A, B, C, and D) as the products to be identified. The choice of products was motivated by the following reasons: the survey suggested that wine is a product predominantly bought in traditional shops and the individual products can be easily identified by name. Also, consumers rely on additional information for wine purchases, such as recommendations from friends and test institutes. The wine bottles were selected randomly in a supermarket from more than 300 different wine bottles offered, in order to achieve a sufficient randomization of the product name. Each of the bottles had an EAN13 barcode attached, and a product name label. In addition, we tagged each bottle with an NFC RFID tag and an EPC RFID tag. The appearance of the bottles is depicted in Fig. 1.

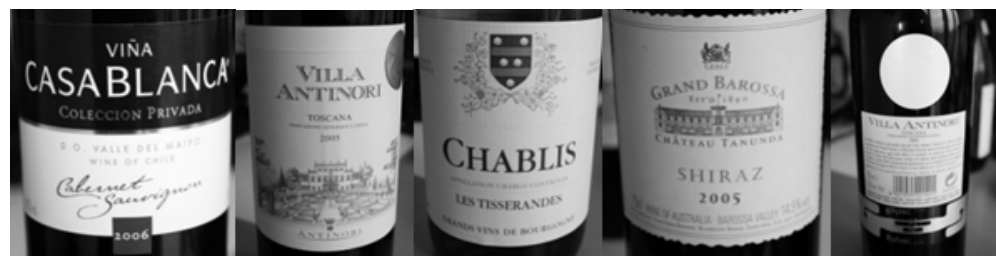

Fig. 1. Labels and tagging of the wine bottles used

The independent variables were the following identification techniques:

Manual Barcode Entry (MBE) denotes the manual entry of the number that is located below a barcode. We used a smart phone (Nokia N95), which is well suited for inputting numbers, with a simple application allowing the user to enter barcodes (EAN8, UPC12, EAN13), pressing a button for confirmation and receiving feedback that the product was identified. In the case of our study, all products were equipped with the 13-digit EAN13 barcodes. These are most commonly used for consumer products.

Manual Product Search (MPS) required the user to enter a search term for the given product into a text field. For this condition the iPhone was used, as it is widely used for accessing the mobile Internet. In contrast to many other phones it offers a fairly large on-screen keyboard. For comparability reasons, we only measured the time the used needed to enter a search term until he pressed the search button. We deliberately did not predefine the term to search for. We assume that one key disadvantage of 
today's practice is that consumers in most cases cannot search for product information in the web based on a tag on the product (an exception might be searching for books, based on the ISBN). Thus we believe that for quantifying the benefit of automatic identification, the time needed to find an appropriate search term should be included in the measurement.

For Automatic Barcode Recognition (ABR) the participants used a smart phone (Nokia N95) with a built-in camera. For the task a simple application based on a barcode recognition toolkit [6] was used. The barcode recognition software used is from our experience the most reliable barcode reader. Users had to scan the barcode by moving the product's barcode into the field of the lens coverage of the handset's camera at a distance of about 3 centimeters. They received feedback when the barcode was recognized.

NFC Tag Scan (NFC) was implemented with a simple Java application using JSR 257 on a Nokia 6131 NFC handset. The latter is a commercially available phone with an integrated NFC RFID reader. Performing the task required the user to touch a tag with mobile phone antenna. The reading range is about $5 \mathrm{~cm}$. The user received a sound feedback as soon as the tag was recognized.

EPC Tag Scan (EPC) was done on a prototype phone (Nokia E61i with integrated EPC UHF RFID reader) using a simple Java application. To identify the product the user had to point the phone towards the product (reading range up to $50 \mathrm{~cm}$, according to the manufacturer).

In the beginning, the participants received an oral introduction (roughly $10 \mathrm{~min}$ utes) to the different identification techniques and were encouraged to try them out. Users tested each technique a couple of times until they confirmed that they felt familiar with each. We briefly motivated the use of product identification techniques on mobile devices with potential application scenarios. After this, the participants completed two tasks. Following the study, we conducted an interview with each of the participants.

For each participant the study was recorded on video. In a later step we extracted the precise time recording for the identification techniques from the video ${ }^{2}$. We also transcribed all comments the participant made during the study.

\subsection{Task 1 - Single Product Identification}

The task for the participants was to use every identification technique (MBE, MPS, ABR, NFC, EPC) on each product (A, B, C, D). In total, each participant performed 20 identification tasks, their order was randomized. Overall, task 1 took about 10 minutes per participant.

Each of the 20 identifications was performed in the following steps: A mobile handset with the software supporting the respective identification technique was handed to the participant. The identification application was active in all cases. As soon as the participant confirmed readiness, the supervisor touched one of the four

\footnotetext{
${ }^{2}$ A video summarizing the study and the results is available at: http://www.youtube.com/watch?gl=DE\&v=d1 AgudZwPkM
} 
bottles (simulating the discovery of an interesting product as described above) in order to indicate for which product the identification task should be performed. Touching the bottle is also used as a trigger to measure time. The participant then identified the product. As soon as the product was identified, time recording was stopped. The mobile phone was returned to a supervisor and reset.

\subsection{Task 2 - Comparison of Multiple Products}

In the second task we compared one manual and one automatic identification technique. We have selected Manual Barcode Entry (MBE) and NFC Tag Scan (NFC), as they have proven fastest and most convenient for the users.

Each participant performed the comparison task (for time reasons) only with three randomly chosen bottles (A, C, and D); once with each technique. Half of the participants began with technique MBE, before performing the task with technique NFC, and vice versa. As in task 1, each participant was given a handset with an active application which allowed identification with the respective technique. After a trigger signal, the participant identified the three bottles in a row. With the confirmation of the last identification (beep) the time recording was stopped.

We measured the time the participants needed for three subsequent identifications using each technique and evaluated all comments made during the experiment by video analysis.

\subsection{Interview}

After having performed the above tasks, we asked each participant to rate each identification technique on a Likert scale from 1 (very hard to use) to 5 (very easy to use). We concluded the interview with an informal discussion about the individual techniques encouraging participants to share their perceptions. Finally, we clarified the concrete intention of the study to the participants.

\section{Usability Study: Results}

The following paragraphs describe the results of the study. In addition to the clearly measurable variables 'task completion time' and 'perceived ease of use', we describe comments made by the participants and observations made during the experiments.

\subsection{Automatic Techniques Up to Eight Times Faster, Barcode Almost as Fast as RF}

Measuring the time per single product identification (task 1) indicates that automatic identification is significantly faster than manual techniques (see Figure 2). While this could have been expected, the experiments reveal that the fastest automatic identification technique (NFC) is roughly eight times faster per identification than the slowest user-mediated identification technique (MPS). From the comments during the study it becomes apparent that manual input is not considered an alternative when searching for product information on mobile phones. Participant P2 highlights this saying "why do you bother to do the test, it is clear nobody's gonna do this". 


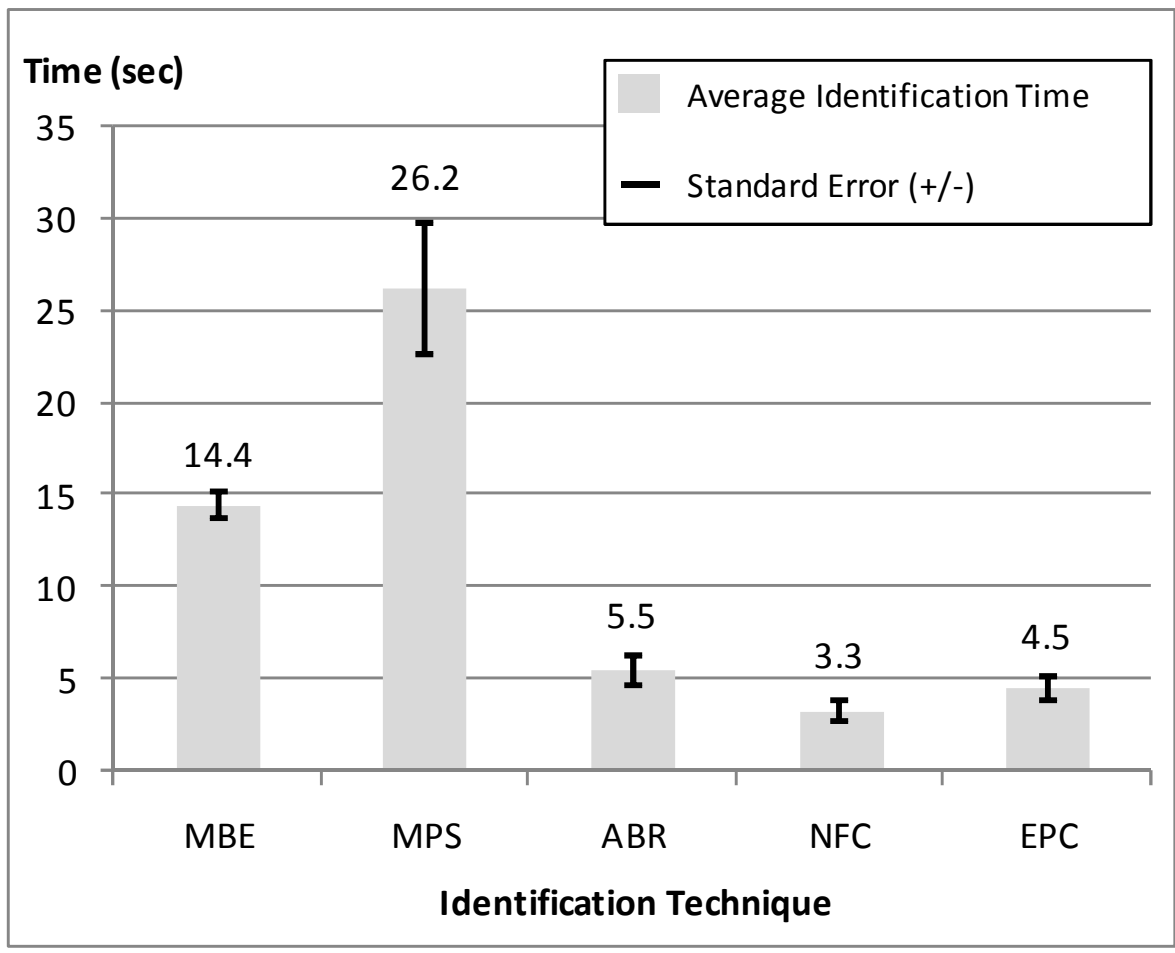

Fig. 2. Average Time per Identification by Technique

The fastest technique in the experiment is the NFC Tag Scan (NFC), taking the users 3.3 seconds in average to identify a product. Scanning NFC Tags is time-wise followed by EPC Tag Scans (EPC), which takes the user in average 4.5 seconds. In this context it is worth mentioning that, at the current technical level, NFC tag scan and the EPC tag scan interact with products in the same way - by touching the tag on the product with the antenna integrated in the phone. Although both RFID-based identification techniques (NFC, EPC) are the fastest, they are, surprisingly, relatively closely followed by the third automatic identification technique, the automatic barcode recognition (ABR, 5.4 seconds on average).

In contrast, manual barcode entry (MBE) takes approximately three times as long as recognizing the barcode automatically. Moreover, the technique that takes the users by far the longest time is entering product information on a search page (MPS), taking about eight times as long as scanning an NFC tag. The large temporal deviations of technique MPS are due to the fact that each participant could freely pick the term considered best suited to find information about the product on hand, leading to search terms of between 7 and 31 characters length. In addition, heavy iPhone users perform slightly better than those who have never used an iPhone before. 


\subsection{Approximately Linear Progression of 'Task Completion Time'}

The time for three identifications in a row (task 2) was performed by means of manual barcode entry (MBE) and NFC tag scan (NFC). Comparing three products can be considered a typical task during a buying decision. While the automatic identification of three products takes 8.4 seconds on average, the manual identification is with 39.2 seconds significantly slower.

\subsection{Correlation between 'Task Completion Time' and 'Perceived Ease of Use'}

The results of the assessment of perceived ease of use are depicted in Figure 3.

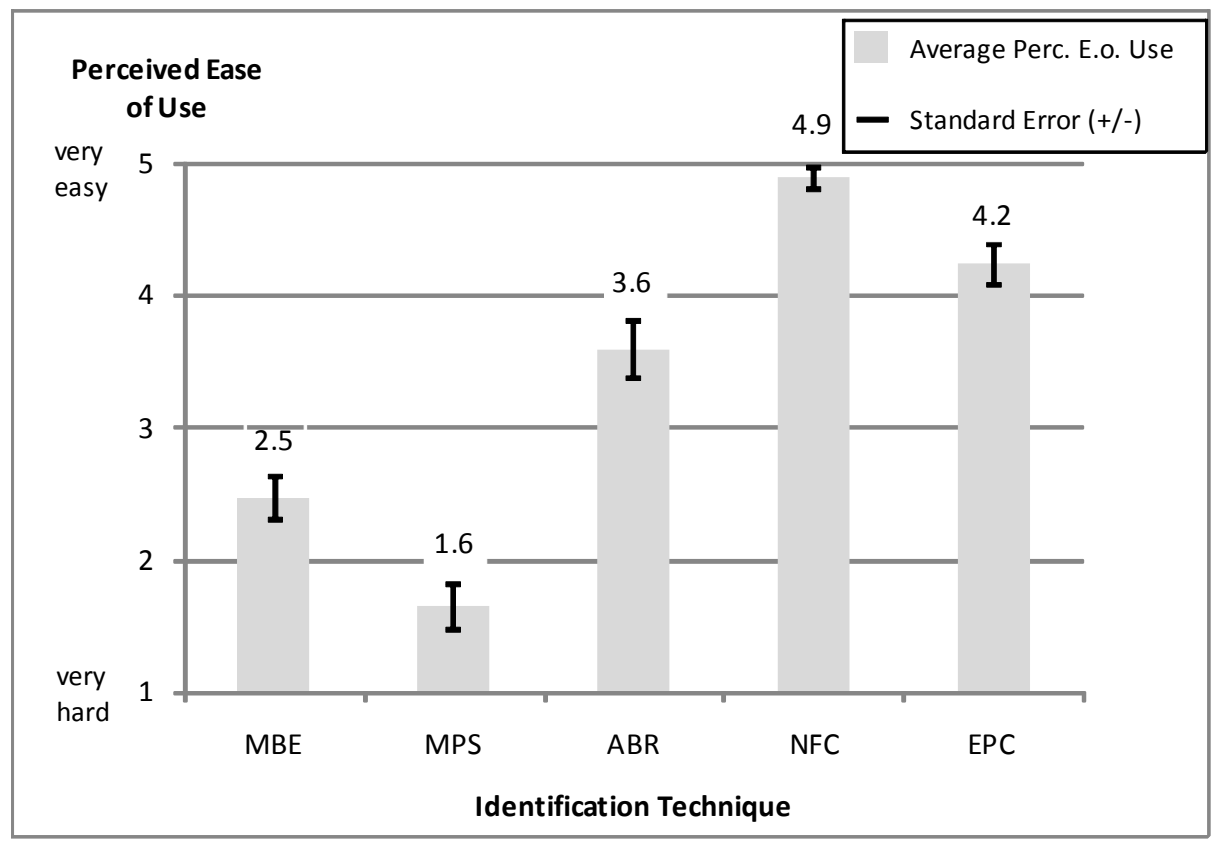

Fig. 3. Average Perceived Ease of Use per Identification Technique

The NFC tag scan (NFC) is perceived as the easiest-to-use identification technique with an average score of 4.9 and only two people not rating it at 5. The EPC tag scan (EPC) is slightly behind (4.2), with some more distance to barcode recognition. The manual identification techniques are according to the user ratings significantly harder to use.

Ordering the identification techniques by speed provides the same result as ranking them by user preference. This indicates a strong correlation between the time needed for identification using a particular technique and the perceived ease of use. Interestingly, three out of six female participants of the study do not perceive a significant difference regarding the ease of use of entering a barcode manually and recognizing it automatically. 


\subsection{Variable Attention Requirements per Technique}

Observing the participants performing the tasks we noticed large differences in the attention a user needs to use the different techniques. In particular identification techniques NFC and EPC can be performed with little effort. Participants stated that they could imagine technique MBE, MPS, and ABR being serious hurdles for people with limited attention capabilities.

\section{Discussion and Conclusion}

Our survey shows that when shopping online, the access to additional information can potentially be valuable to consumers. The mobile phone offers possibilities to provide this information. More specifically, the survey shows what information consumers exactly base their buying decision on. Given these results, applications should provide easy mobile access rather to technical details and price comparisons for so called 'search products', and to recommendations of friends and family members and own experiences (for example with wine) for so called 'experience products'. To our knowledge, the survey is the first of its kind providing figures on the motivation of consumers to pay for additional product information on mobile phones. Roughly $70 \%$ of the consumers are not willing to pay for additional product information on their mobile phone. Consequently, entrepreneurs will have to think about appropriate business models for mobile product information systems. If the consumer does not pay, who else will pay? Our experience shows that for example retailers fear the transparency generated through mobile shopping assistants, but at the same time they gradually discover that it will be difficult to prevent consumers from using such systems. While our survey has provided insights for the given respondents group, future work will also have to investigate whether the results are different for the so called 'ringtone' generation below the age of 20, who have been underrepresented in our survey, but provide a target group for mobile services.

Our user study indicates a significant advantage of automatically recognizing an identifier compared to entering a search term or a 13-digit number in a text field (both are the only ways to interact with products using a mobile phones that are applied today). This lets us assume that product-related services will not widely be used as long as automatic identification is not available on mobile phones. A frequently disregarded issue is that automatic identification is even more important when interacting with more than one product, as simulated in task 2 . While consumers might scan three products in a row ( 8.3 seconds) it is hard to believe that anybody will enter three barcodes manually (39.2 seconds).

Our figures show that barcode recognition can be time-wise performed almost as fast as scanning RFID tags, using one of the best mobile barcode scanners available. This fact, in combination with the pervasiveness of barcodes among today's products, makes the detection of barcodes a promising mobile identification technique in today's world, at least as long as the consumer goods industry does not switch to RFID on item-level. In addition, our results show that algorithms for detecting barcodes should take significantly less time than the 14.4 seconds the manual entry of a barcode takes in average. Given the differences in the performance of mobile barcode 
recognition today, this is not self-evident. A further point worth mentioning is that at current technical level, there is no barcode reader that works for a broad range of mobile platforms and handsets.

On a more abstract level we conclude that, despite the number of already commercially available mobile product-related services based on automatic identification, it needs to be worked on fast, reliable, and broadly applicable barcode readers for the identification of current products. Manual product identification as applied for example on the iPhone today (to our knowledge there is no $1 \mathrm{~d}$ barcode reader on the iPhone that works without an additional lens on the camera) cannot be considered an alternative. In addition, novel revenue models have to be developed as a majority of consumers is not willing to pay for product information on their mobile phone.

As a next step we will propose an architecture for invoking different services from mobile phones, based on identified products.

\section{Acknowledgements}

This work has been partly funded by the Nokia NRC Pervasive Communications Laboratory in Lausanne (Switzerland), by the research project SemProM (funded by the German Government), and by the Auto-ID Labs initiative.

\section{References}

1. Szymanski, M., Hiseb, R.T.: E-Satisfaction: An Initial Examination. Journal of Retailing 76(3), 309-322 (2000)

2. Senecal, S., Nantel, J.: The Influence of Online Product Recommendations on Consumers' Choices. Journal of Retailing 80(2), 159-169 (2004)

3. Cassar, K. (2008): The Online and In-Store Crossover Conundrum. In: Nielsen Consumer Insight Magazine (2008),

http: / /www.nielsen.com/consumer_insight/issue10/ci_story1.html (checked January 2008)

4. Ward, M.R., Morganowsky, M.: Consumer Acquisition of Product Information and Subsequent Purchase Channel Decisions. The Economics of the Internet and E-Commerce 11, 231-255 (2002)

5. Rohs, M., Gfeller, B.: Using Camera-Equipped Mobile Phones for Interacting with RealWorld Objects. In: Ferscha, A., Hoertner, H., Kotsis, G. (eds.) Advances in Pervasive Computing, pp. 265-271. Austrian Computer Society (OCG) (2004)

6. Adelmann, R.: Mobile Phone Based Interaction with Everyday Products - On the Go. Conference on Next Generation Mobile Applications, Services and Technologies (2007)

7. NFC Forum NFC Data Exchange Format Technical Specification (2008), http: / / www. nfC-forum. org

8. Ondrus, J., Pigneur, Y.: An Assessment of NFC for Future Mobile Payment Systems. In: Conference on the Management of Mobile Business (2007)

9. Anokwa, Y., Borriello, G., Pering, T., Want, R.: A User Interaction Model for NFC Enabled Applications. Pervasive Computing and Communications Workshops (2007)

10. Geven, A., Strassl, P., Ferro, B., Tscheligi, M., Schwab, H.: Experiencing Real-World Interaction: Results from a NFC User Experience Field Trial. In: Conference on Human Computer Interaction with Mobile Devices and Services (2007) 
11. Savolainen, J., Iraji, S., Hirvola, H.: EPC UHF RFID Reader: Mobile Phone Integration and Services. In: Consumer Communications and Networking Conference (2009)

12. Thiesse, F., Michahelles, F.: An Overview of EPC Technology. Sensor Review 26(2), 101-105 (2006)

13. Sarma, S.: Towards the $5 \varnothing$ Tag, Massachussets Institute of Technology, Auto-ID Center White Paper (2001)

14. Wiechert, T., Thiesse, F., Michahelles, F., Schmitt, P., Fleisch, E.: Connecting Mobile Phones to the Internet of Things: A Discussion of Compatibility Issues between EPC and NFC. In: Americas Conference on Information Systems, AMCIS (2007)

15. von Reischach, F., Guinard, D., Michahelles, F., Fleisch, E.: A Mobile Product Recommendation System Interacting with Tagged Products. In: IEEE Conference on Pervasive Computing and Communications, PerCom 2009 (2009)

16. O’Hara, K., Perry, M.: Shopping Anytime Anywhere. In: CHI Extended Abstracts on Human Factors in Computing Systems (2001)

17. Resatsch, F., Sandner, U., Leimeister, J.M., Krcmar, H.: Do Point of Sale RFID-based Information Services Make a Difference? Analyzing Consumer Perceptions for Designing Smart Product Information Services in Retail Business. Electronic Markets 18(3), 216-231 (2008)

18. Zenebe, A., Ozok, A., Norcio, A.F.: Personalized Recommender Systems in E-commerce and M-commerce: A Comparative Study. In: Conference on Human-Computer Interaction (HCI International) (2005)

19. Häubl, G., Trifts, V.: Consumer Decision Making in Online Shopping Environments: The Effects of Interactive Decision Aids, Marketing Science (2000)

20. Ballagas, R., Borchers, J., Rohs, M., Sheridan, J.: The Smart Phone: A Ubiquitous Input Device. IEEE Magazine on Pervasive Computing 5(1), 70-77 (2006)

21. Broll, G., Siorpaes, S., Rukzio, E., Paolucci, M., Hamard, J., Wagner, M., Schmidt, A.: Comparing Techniques for Mobile Interaction with Objects from the Real World. In: Permid Workshop at the Pervasive Conference (2007)

22. Rukzio, E., Broll, G., Leichtenstern, K., Schmidt, A.: Mobile Interaction with the Real World: An Evaluation and Comparison of Physical Mobile Interaction Techniques. In: Conference on Ambient Intelligence, AmI (2007)

23. Nelson, P.: Information and Consumer Behavior. Journal of Political Economy 78(2), 311 $329(1970)$

24. King, M.F., Balasubramanian, S.K.: The Effects of Expertise, End Goal, and Product Type on Adoption of Preference Formation Strategy. Journal of the Academy of Marketing Science 22(2), 146-159 (1994) 